\title{
Facteurs culturels liés au maintien des comportements de santé chez des femmes algonquines présentant des antécédents de diabète gestationnel
}

\author{
S. Gaudreau, M. Sc. (1); C. Michaud, Ph. D. (2)
}

Cet article a fait l'objet d'une évaluation par les pairs.

\section{Résumé :}

Introduction : Les facteurs culturels qui pourraient contribuer à l'épidémie de diabète chez les Premières nations ont souvent été présentés, mais les facteurs qui pourraient participer à sa prévention demeurent peu connus. La présente étude ethnoinfirmière explore les facteurs culturels qui favorisent le maintien des comportements de santé chez des femmes algonquines ayant reçu un diagnostic de diabète gestationnel 2 à 10 ans avant le début de l'étude.

Méthodologie : Les données ont été recueillies dans deux communautés algonquines par le biais d'entrevues semi-dirigées auprès d'informatrices-clés ( $\mathrm{n}=7$ ) et d'informateurs généraux $(n=8)$ ainsi que grâce à une immersion culturelle avec des observations détaillées inscrites dans un journal de bord.

Résultats : Les facteurs culturels qui pourraient avoir une influence sur la prévention du diabète sont l'importance de la famille et des liens sociaux, la possibilité de conserver ses valeurs culturelles, la possibilité d'adopter de nouveaux comportements à l'aide de ressources éducatives adaptées à ses besoins et à sa culture, la possibilité de réaliser des économies grâce à une meilleure alimentation, et l'accès à ses données de glycémie comme moyen de contrôle.

Conclusion : À long terme, ces facteurs culturels pourraient influencer les comportements de santé et, ainsi, aider à prévenir le diabète de type 2 .

Mots-clés : facteurs culturels, diabète gestationnel, femmes algonquines, comportements de santé, santé, diabète de type 2.

\section{Introduction}

Le diabète gestationnel (DG) est une intolérance au glucose qui apparaît pour la première fois durant la grossesse $\mathrm{e}^{1}$ et qui disparaît généralement après l'accouchement ${ }^{2}$. Le DG est associé à des complications comme une macrosomie, une hypertension gravidique et une prééclampsie, qui entraînent des risques pour la mère et son enfant à l'accouchement ${ }^{3-4}$. La moitié des femmes ayant reçu un diagnostic de DG développeront un diabète de type 2 au cours de leur vie ${ }^{2,5,6}$. Les interventions destinées aux femmes atteintes de DG comprennent l'accompagnement en vue de l'adoption de comportements de santé qui permettront de maintenir une glycémie normale et, par le fait même, de réduire le risque de complications pour la mère et son enfant ${ }^{1,4,5,7}$. Or, ces interventions doivent être dispensées dans un contexte culturel et dans le respect de leur signification culturelle ${ }^{8-10}$; on définit ici le concept de culture comme "les valeurs, les croyances, les normes et les pratiques de mode de vie d'un groupe donné qui sont apprises, partagées et transmises, et façonnent de façon structurée la manière dont les personnes pensent, prennent des décisions et agissent »(traduction libre) ${ }^{9}$.

De nombreux chercheurs ont étudié le diabète, principalement de type 2 , ainsi que les dimensions culturelles au sein des Premières nations et d'autres populations autochtones américaines ${ }^{10-15}$. Selon ces études, le diabète serait perçu comme une " maladie de Blancs " par ces communautés ${ }^{11-13}$, et il serait indissociable des profondes transformations socioéconomiques et politiques qui ont marqué les relations entre les sociétés autochtones et non autochtones ${ }^{12}$. En effet, la transformation du mode de vie traditionnel des Autochtones aurait créé des inégalités socioéconomiques ayant des répercussions directes sur la santé (p. ex., moins de richesses naturelles disponibles, consommation moindre d'aliments traditionnels et accessibilité limitée à des aliments sains et nutritifs dans certaines communautés) ${ }^{15}$. Par ailleurs, les Premières nations auraient des conceptions différentes de la santé, notamment une conception fataliste du diabète ${ }^{14}$. La nourriture serait considérée comme un signe d'hospitalité et la minceur pourrait être considérée comme péjorative ${ }^{13}$, tout comme l'activité physique, par exemple la marche ${ }^{11,12}$. Enfin, les différences culturelles ne seraient pas prises en compte dans les

Rattachement des auteurs :

1. Centre de santé et de services sociaux Les Eskers de l'Abitibi, Amos (Québec), Canada

2. École des sciences infirmières, Faculté de médecine et des sciences de la santé, Université de Sherbrooke, Longueuil (Québec), Canada

Correspondance : Cécile Michaud, École des sciences infirmières, Faculté de médecine et des sciences de la santé, Université de Sherbrooke, Campus de Longueuil, 150, place Charles-Le Moyne, bureau 200, Longueuil (Québec) J4K 0A8; tél. : 450-463-1835 poste 61793; téléc. : 450-463-1837; courriel : Cecile.Michaud@USherbrooke.ca 
soins conçus pour modifier le mode de vie ${ }^{13}$. Tous ces facteurs culturels constitueraient des obstacles aux comportements de santé.

Dans le cadre de la présente étude qualitative, nous avons cherché à comprendre les facteurs culturels qui contribuent au maintien des comportements de santé encouragés pendant la grossesse chez des femmes algonquines ayant reçu un diagnostic de DG. Cette compréhension permettrait d'offrir des soins compétents sur le plan culturel, visant à préserver les comportements qui maintiennent la santé de ces femmes, ainsi que celle de leurs enfants et de leur entourage.

\section{Méthodologie}

L'approche ethnographique vise à mieux comprendre un système culturel du point de vue de ceux qui partagent cette culture ${ }^{16}$. L'approche ethnoinfirmière que nous avons adoptée permet d'expliquer le mode de vie et la vision culturelle d'un individu tout en tenant compte du contexte. Elle vise à décrire, à comprendre et à interpréter la signification des pratiques, des croyances et des valeurs d'autres cultures $8,10,17$.

\section{Population}

Deux communautés algonquines (Pikogan et Lac Simon*) ont été choisies pour cette étude, en raison de leur proximité géographique et de l'intérêt des directrices des centres de santé envers l'étude. Pikogan est en milieu urbain, tandis que
Lac Simon est en milieu rural. Les deux communautés sont toutes deux principalement francophones, bien que la langue algonquine y subsiste toujours. Pikogan est un village situé à environ $3 \mathrm{~km}$ de la municipalité d'Amos. En 2008, 278 de ses 551 habitants inscrits étaient des femmes ${ }^{18}$. Environ $52 \%$ de la population avait moins de 25 ans $^{19}$. La communauté de Lac Simon est située à environ $32 \mathrm{~km}$ de la municipalité de Val-d’Or. En 2008, 659 de ses 1362 habitants inscrits étaient des femmes ${ }^{18}$, et environ $61 \%$ de la population était âgée de moins de 25 ans $^{19}$. Les deux communautés possèdent des services sanitaires, éducatifs et communautaires similaires, soit un centre de santé, des services de médecins visiteurs, une école primaire, une garderie, un poste de police, une église, une salle communautaire, un dépanneur, une radio communautaire, une maison de jeunes et les édifices appartenant à chacun des Conseils de bande respectifs. Il y a de plus une école secondaire, un bureau de poste et un restaurant à Lac Simon, tandis que la communauté de Pikogan dispose d'un aréna.

\section{Sélection des informateurs et recrutement}

Une étude ethnoinfirmière s'appuie sur l'information fournie par deux types de participants : les informateurs-clés et les informateurs généraux ${ }^{8,9}$. Les informateursclés sont directement concernés par la problématique; dans le cas présent, il s'agissait de femmes $(n=7)$ ayant reçu un diagnostic de DG ainsi que des soins au centre de santé de leur communauté (tableau 1). Les critères d'inclusion suivants ont été employés : origine algonquine, femmes de 18 ans ou plus, diagnostic de DG reçu entre 2 et 10 ans avant le début de l'étude, femmes ayant reçu des soins de santé dans la communauté algonquine à la suite du diagnostic de DG, femmes qui n'étaient pas enceintes ou qui n'allaitaient pas durant l'étude. Les informatrices-clés ont été recrutées par trois des informateurs généraux.

Pour des raisons d'anonymat, l'âge et le niveau de scolarité de chacune des informatrices-clés ne sont pas précisés; cependant, l'âge moyen était de 34 ans (29-40 ans), et la moitié des informatricesclés n'avaient pas obtenu leur diplôme d'études secondaires. Nous avons attribué un prénom fictif à chacune des informatricesclés, semblable aux prénoms en usage dans les deux communautés et attribué selon l'ordre des entrevues. Ainsi, la première personne interrogée à Lac Simon s'est vu attribuer la lettre « $\mathrm{A}$ » ainsi qu'un prénom commençant par cette lettre (p. ex. Amy), et ainsi de suite jusqu'à la lettre " $\mathrm{D}$ ». Pour les différencier, les femmes de Pikogan se sont vues attribuer des prénoms fictifs en commençant par la lettre «I », pour terminer par la lettre « $\mathrm{K}$ » (voir le tableau 1).

Les informateurs généraux ont quant à eux une idée plus générale de la problématique. Ils nous ont été présentés par les personnes-ressources qui nous

TABLEAU 1

Résumé du profil sociodémographique et sanitaire des informatrices-clés $(n=7)$

\begin{tabular}{|c|c|c|c|c|c|c|c|c|}
\hline \multirow[t]{2}{*}{ Prénom fictif ${ }^{a}$} & \multirow[t]{2}{*}{ Communauté } & \multicolumn{3}{|c|}{ Langue } & \multirow{2}{*}{$\begin{array}{c}\text { Diagnostic de } \\
\text { DG }\end{array}$} & \multirow{2}{*}{$\begin{array}{c}\text { Diagnostic de } \\
\text { DT2 }\end{array}$} & \multirow[t]{2}{*}{ IMC, $\mathrm{kg} / \mathrm{m}^{2}$} & \multirow{2}{*}{$\begin{array}{l}\text { Nombre } \\
\text { d'enfants }\end{array}$} \\
\hline & & Algonquin & Français & Anglais & & & & \\
\hline Amy & Lac Simon & Oui & Oui & Non & 1999 & Oui & 36,0 & 6 \\
\hline Brenda & Lac Simon & Non & Oui & Non & 2003 & Oui & 28,3 & 6 \\
\hline Céline & Lac Simon & Non & Oui & Non & 2004 & Non & 36,0 & 5 \\
\hline Diane & Lac Simon & Oui & Oui & Non & 2003 & Non & 34,4 & 4 \\
\hline Isabelle & Pikogan & Non & Oui & Oui & 2003 & Non & 33,9 & 4 \\
\hline Julie & Pikogan & Non & Oui & Oui & 2003 & Non & 36,6 & 5 \\
\hline Kimberly & Pikogan & Non & Oui & Non & 2003 & Non & 28,9 & 5 \\
\hline
\end{tabular}

Abréviations : DG, diabète gestationnel; DT2, diabète de type 2; IMC, indice de masse corporelle.

${ }^{a}$ Un prénom fictif, semblable aux prénoms en usage dans les deux communautés, a été attribué à chacune des informatrices-clés, selon l'ordre des entrevues. Ainsi, la première personne interrogée à Lac Simon s'est vu attribuer la lettre « A » ainsi qu’un prénom commençant par cette lettre, et ainsi de suite jusqu’à la lettre « D ». Pour différencier les femmes de Pikogan, nous avons commencé l'attribution des prénoms fictifs à la lettre « $\mathrm{I} »$, pour terminer par la lettre « $\mathrm{K} »$.

* Les directrices des deux centres de santé communautaires concernés ont accepté que le nom des communautés soit divulgué dans le présent article. 
servaient de premiers interlocuteurs avec les communautés à l'étude. Les informateurs généraux ont accepté de parler librement de leurs expériences dans la communauté et de fournir des renseignements supplémentaires sur les observations faites. En tout, huit personnes ont été consultées à titre d'informateurs généraux. Il s'agissait de 4 infirmières, d'une nutritionniste, d'un travailleur social, d'un infirmier auxiliaire et d'une hygiéniste dentaire. Deux d'entre eux étaient issus des Premières nations et la plupart $(n=6)$ travaillaient à Lac Simon. Leur expérience de travail dans les communautés concernées variait de quelques mois à huit ans ( $\bar{x}=3,5$ ans).

\section{Collecte des données}

Les données ont été recueillies par observation participante et entrevues semi-dirigées. Une immersion culturelle de 2 mois dans les deux communautés a eu lieu en mars et en avril 2006, ce qui nous a permis d'observer les activités des intervenants de la santé de Lac Simon et de Pikogan, et de participer à des cuisines collectives, des repas communautaires, des visites à domicile, etc. En outre, nous avons pu noter certains aspects de l'environnement physique, comme le type d'aliments offerts par le dépanneur et le club des petits déjeuners. Nous avons consigné nos observations dans un journal de bord, dans un format condensé et homogène encourageant la réflexion, comme le recommande l'outil Observation-Participation-Reflection ${ }^{8}$. Des entrevues semi-dirigées ont été réalisées en français auprès des informatrices-clés, généralement au centre de santé communautaire. Ces entrevues étaient fondées sur les facteurs culturels de Leininger ${ }^{8,9}$ et l'étude de Taylor et collab. ${ }^{14}$ (voir l'annexe A); d'une durée moyenne de 47 minutes, elles ont été enregistrées afin de permettre par la suite de les transcrire intégralement et de les analyser.

Il convient de noter que les entrevues portaient sur le maintien des comportements de santé, et non sur les obstacles à ce maintien. En outre, la présente étude ne visait pas à influencer ni à juger les comportements de santé existants.

\section{Analyse des données}

L'analyse des données d'observations a été effectuée en quatre phases ${ }^{10}$, et ce, en suivant la démarche de Miles et Huberman ${ }^{20}$. $\mathrm{Au}$ cours de la première phase, qui a été alimentée par les observations réalisées lors de l'immersion culturelle et les entrevues auprès des femmes algonquines, le journal de bord a été utilisé pour mieux replacer le sens des verbatims dans leur contexte. Lors de la deuxième phase, nous avons procédé à l'analyse verticale du verbatim des entrevues en classant chaque phrase selon un des facteurs culturels de Leininger $^{8,9}$, afin de faire ressortir tous les facteurs culturels mentionnés dans chaque transcription. La troisième phase, celle de l'analyse des profils et du contexte, consistait quant à elle en une analyse horizontale des facteurs culturels, ce qui a permis de regrouper les facteurs culturels ayant contribué au maintien des comportements de santé encouragés durant la grossesse chez toutes les participantes. La quatrième et dernière phase prévoyait pour sa part la confirmation des thèmes et sous-thèmes par les informatrices-clés et les informateurs généraux. Un seul thème a été reconstruit après une telle confirmation : l'importance des amis dans le maintien des comportements de santé.

L'annexe B présente les stratégies employées afin d'assurer la rigueur de l'analyse de données.

\section{Considérations éthiques}

La présente étude a été approuvée par le comité d'éthique du Centre de recherche clinique du Centre hospitalier universitaire de Sherbrooke et par les directrices des centres de santé de Pikogan et de Lac Simon. Elle respecte les lignes directrices de l’Énoncé de politique des trois Conseils ${ }^{21}$ relatives aux projets de recherche menés sur des terres appartenant aux Premières nations. Par ailleurs, nous avons au préalable obtenu l'autorisation écrite des responsables des communautés, et nous avons attribué des noms fictifs aux participantes, tel que décrit précédemment, afin de préserver la confidentialité des données.

\section{Résultats et analyse}

Le tableau 2 résume les principaux facteurs culturels qui ont contribué au maintien des comportements de santé; ces facteurs sont classés en fonction des cinq thèmes et des différents sous-thèmes identifiés. Les résultats sont présentés et analysés dans la même section.

\section{La famille et les liens sociaux comme motivation et soutien pour le maintien des comportements de santé}

La famille et les liens sociaux constituaient la principale motivation au maintien des comportements de santé. Tout comme dans l'étude de Taylor et collab. ${ }^{14}$, les femmes algonquines ne souhaitaient pas que leurs enfants développent à leur tour un diabète : « Oui, j’ai gardé ma manière de comment je mangeais. J'ai gardé ça surtout concernant moi puis ma fille. Je ne veux pas qu'elle ait du diabète d'adolescence, elle non plus. Elle a un surplus de poids, puis je ne veux pas ça pour elle. Fait que j'ai gardé ça... mais plus pour moi... puis elle aussi » (Julie', ligne 528).

De plus, les expériences des parents des informatrices-clés faisaient office de rappel constant des complications possibles de la maladie : "Je ne peux pas avoir des complications déjà dans 5 ans... Mon père a fait de la dialyse, puis ma grand-mère s'est fait amputer » (Amy, ligne 45).

Le soutien de l'entourage et de la communauté occupait une place importante. Ce soutien pouvait se traduire par des encouragements : "Tout le monde me dit que ça paraît que tu es en train de perdre du poids. Puis ça aide aussi quand quelqu'un te dit ça » (Julie, ligne 840). Il pouvait aussi prendre la forme d'un accompagnement : " $[\ldots]$ on essayait de faire une sorte de régime de ce qu'on pourrait manger, moi puis elle, pour soutenir notre diabète » (Julie, ligne 359). L'entourage donnait également des conseils : " [Ma sœur] me disait : "Ça, il y a trop de sucre. Dans ça, il y a ça. Combien de calories il y a dans un repas, si tu vas au restaurant?". Parce que ma sœur fait du diabète. C'est ça qui m’a aidé beaucoup aussi » (Kimberly, ligne 378). 
TABLEAU 2

Facteurs favorisant le maintien des comportements de santé chez les informatrices-clés $(\mathbf{n}=7)$

\begin{tabular}{|c|c|c|}
\hline Facteurs & Thèmes & Sous-thèmes \\
\hline $\begin{array}{l}\text { Facteurs familiaux } \\
\text { et sociaux }\end{array}$ & $\begin{array}{l}\text { La famille et les liens sociaux comme motivation } \\
\text { et soutien pour le maintien des comportements } \\
\text { de santé }\end{array}$ & $\begin{array}{l}\text { Préoccupation au sujet de la santé des enfants } \\
\text { Soutien de l'entourage et de la communauté } \\
\text { Expériences des membres de la famille } \\
\text { Désir d'être ensemble }\end{array}$ \\
\hline $\begin{array}{l}\text { Mode de vie et } \\
\text { valeurs culturelles }\end{array}$ & $\begin{array}{l}\text { Adopter de nouveaux comportements de santé tout } \\
\text { en conservant ses valeurs culturelles }\end{array}$ & $\begin{array}{l}\text { Adaptation de l'alimentation traditionnelle } \\
\text { Modification de l'alimentation } \\
\text { Pratique de la marche } \\
\text { Synergie entre l'alimentation et la marche }\end{array}$ \\
\hline $\begin{array}{l}\text { Facteurs } \\
\text { éducationnels }\end{array}$ & $\begin{array}{l}\text { Apprendre à l'aide de ressources éducatives adaptées } \\
\text { à ses besoins et à sa culture }\end{array}$ & $\begin{array}{l}\text { Capacité d'être autodidacte } \\
\text { Adaptation culturelle de l'enseignement et du soutien }\end{array}$ \\
\hline $\begin{array}{l}\text { Facteurs } \\
\text { économiques }\end{array}$ & $\begin{array}{l}\text { Réaliser des économies grâce à une meilleure } \\
\text { alimentation }\end{array}$ & \\
\hline $\begin{array}{l}\text { Facteurs } \\
\text { technologiques }\end{array}$ & $\begin{array}{l}\text { Accéder à ses données de glycémie grâce au } \\
\text { glucomètre }\end{array}$ & $\begin{array}{l}\text { Le glucomètre comme moyen de vérifier immédiatement la normalité de la glycémie } \\
\text { Le téléchargement élimine le besoin de prendre des notes }\end{array}$ \\
\hline
\end{tabular}

Les communautés appuyaient certaines activités physiques, principalement la pratique de la marche, en lui accordant une valeur positive : «Bien, je pense que ça commence à être bien vu [...]» (Kimberly, ligne 939). La marche permettait de socialiser tout en pratiquant une activité physique : «J'y vais avec une amie. On parle, on jase en marchant... des fois avec mon chum » (Kimberly, ligne 947).

Le soutien familial ou social était très important pour les participantes; en l'absence d'un tel soutien, certaines femmes diabétiques des Premières nations trouvent plus difficile d'adopter de manière efficace de nouveaux comportements de santé12. D'un point de vue psychosocial, les gens soutenus par leurs pairs sont moins susceptibles de développer des problèmes de santé d'ordre physique ou mental ${ }^{22}$.

\section{Adopter de nouveaux comportements de santé tout en conservant ses valeurs culturelles}

Le deuxième thème porte sur le fait d'adopter de nouveaux comportements de santé, notamment en matière d'alimentation et d'activité physique, tout en conservant ses valeurs culturelles.

Selon les femmes algonquines, leur alimentation traditionnelle peut être adaptée afin de favoriser le maintien des comportements de santé. La méthode de cuisson de certains aliments traditionnels ainsi que l'utilisation de moins de matières grasses dans la préparation des mets pourraient contribuer à une bonne santé et à la prévention du diabète. Les informatricesclés ont aussi mentionné pouvoir privilégier certaines viandes sauvages moins grasses (p. ex. opter pour l'orignal plutôt que le castor).

Les informatrices-clés ont modifié leur alimentation après avoir reçu un diagnostic de DG, et leurs nouvelles habitudes alimentaires équilibrées reflétaient les objectifs fixés par le Guide alimentaire canadien ${ }^{23}$. Les modifications consistaient surtout à compléter les aliments traditionnels, et non à bannir ces derniers. En raison de leur aspect symbolique, l'utilisation des aliments et des méthodes de préparation traditionnels revêt une grande importance pour les membres des Premières nations, qui se sentent acceptés et respectés lorsque les professionnels de la santé tiennent compte de ces pratiques traditionnelles ${ }^{24}$.

Les informatrices-clés ont manifesté le désir d'avoir accès à un livre de recettes qui serait conçu pour les aider à prendre en charge leur diabète et inclurait des mets traditionnels, ce qui valoriserait le respect de leurs valeurs traditionnelles. Dans les deux centres de santé, des dîners communautaires étaient organisés afin de faire déguster des mets traditionnels adaptés, comme la banik (pain traditionnel) préparée avec de la farine de blé entier, et d'en distribuer les recettes.

Les participantes étaient d'avis que l'exercice, en plus d'une bonne alimentation, est primordial dans le maintien d'un poids santé et la prévention du diabète de type 2 : «Moi, c'est les exercices parce que quand tu t'entraînes beaucoup là, ton alimentation, ça va venir aussi. Je sais que quand je marchais beaucoup, j'avais moins tendance à manger des chips et boire de la liqueur » (Kimberly, ligne 881). La marche était l'activité physique la plus souvent mentionnée dans les entrevues. Associée à de nombreux avantages, la marche permet entre autres de faire le plein d'énergie et de se détendre : «Marcher? Oui, ça m’aide aussi avec le stress à cause je travaille puis j'ai 5 enfants en bas de 10 ans à la maison » (Julie, ligne 746). La pratique de la marche peut aussi être compatible avec les valeurs familiales (p. ex. marcher avec les enfants) et avec les modes de vie des participantes (p. ex. le fait de ne pas avoir d'automobile ou de travailler à l'extérieur de la maison).

L'importance de la perte de poids, décrite par les participantes, est appuyée par des données scientifiques indiquant que l'embonpoint et l'inactivité physique sont des facteurs de risque du diabète de type $2^{2}$ et qu'une perte de poids de 5 à $10 \%$ attribuable à une modification de 
l'alimentation et à la pratique d'une activité physique peut prévenir ou retarder l'apparition d'un diabète de type 2 chez certaines personnes présentant une intolérance au glucose $\mathrm{e}^{25}$. À cet égard, la façon dont les participantes percevaient la minceur et le surpoids était déterminante. En effet, même si toutes les participantes présentaient un indice de masse corporelle (IMC) élevé ( $\bar{x}=33,4)$, elles n'associaient pas la minceur à la maladie, contrairement à d'autres personnes dans d'autres études ${ }^{13}$. Elles avaient plutôt une perception négative du surpoids, tout comme les femmes des Premières nations de l'Oklahoma ${ }^{14}$.

\section{Apprendre de nouveaux comportements à l'aide de ressources éducatives adaptées à ses besoins et à sa culture}

Selon les informatrices-clés, certaines méthodes d'acquisition de nouvelles connaissances offrent davantage de choix et de liberté; ainsi, le troisième thème aborde la possibilité d'apprendre à l'aide de ressources éducatives adaptées à ses besoins et à sa culture. En tant que moyen d'auto-apprentissage, la lecture, par exemple, permet d'éviter des déplacements fréquents au Centre de santé et fournit une certaine forme d'autonomie dans un contexte de pénurie de ressources : "plus souvent aussi ça m’évitait de me déplacer... parce que... vu qu'ils n'en donnaient pas ici des sessions de formation sur ça, puis souvent fallait attendre longtemps avant de voir l'infirmière " (Céline, ligne 1168). La capacité d'être autodidacte et de s'informer favorise le maintien de la santé : " Est-ce qu'il y a des choses que tu fais pour dire “ça, c'est important pour moi pour me maintenir en santé?" (Sylvie, ligne 1183, entrevue avec Isabelle). Oui, m’informer, c'est tout! " (Isabelle, ligne 1187). Cette conclusion concorde avec les observations de Daniel et Messer $^{26}$ selon lesquelles les membres des Premières nations accordent beaucoup d'importance à l'autonomie et pourraient même se méfier des initiatives d'éducation à la santé, les percevant comme une intrusion occidentale dans leur mode de vie. Le modèle de prise de décision partagée ${ }^{27}$ permet d'appuyer cette autonomie; il peut s'avérer plus acceptable qu'un cours magistral sur le diabète.
Les femmes algonquines ayant participé à l'étude ont mentionné l'importance d'apprendre à l'aide de ressources éducatives adaptées à leurs besoins et à leur culture, notamment à leur mode de vie. Elles désiraient par exemple en apprendre davantage sur les ingrédients à privilégier afin de préserver ou d'améliorer la valeur nutritive des mets traditionnels. « J'en fais du banik chez moi là avec de l'huile végétale. Je voulais changer d'huile, mais je ne suis pas certaine laquelle... le moins de gras (le moins pire [sic] pour la santé) » (Julie, ligne 618). Les participantes ont aussi exprimé le besoin de recevoir un enseignement qui respecte le mode de vie traditionnel des Algonquins, notamment le fait qu'ils sont souvent dans les bois : «Qu'est-ce qu'on mange nous autres, les Anicinabek [Algonquins]?...[rires]... Puis comment... on est souvent dans le bois aussi... puis... ils devraient penser à ça... on va dans le bois, là » (Brenda, ligne 855).

Cette adaptation culturelle de l'enseignement peut également se faire par d'autres activités adaptées. Plusieurs informatrices-clés ont parlé d'un "camp diabétique » en forêt, animé par l'infirmière et la nutritionniste, qui intègrent ainsi leur enseignement aux activités traditionnelles (p. ex. promenade dans les bois, excursion en raquettes, etc.). Macaulay et collab. ${ }^{28}$ ont aussi saisi l'importance d'adapter l'enseignement à la culture autochtone (mohawk, dans leur cas) afin de favoriser la prévention du diabète.

Selon les informatrices-clés, le travail constant des professionnels de la santé, en matière de prévention des maladies et de promotion de la santé, constituait un aspect clé dans le maintien des comportements de santé. Les professionnels de la santé encourageaient souvent les personnes ayant fait des progrès sur le plan de la santé, surtout les personnes vivant avec un diabète (quel qu'en soit le type). Ils profitaient aussi des événements rassemblant les membres de la communauté pour mener leurs activités de promotion de la santé. En adaptant les consultations à la façon de vivre des Algonquins, c'est-à-dire en privilégiant l'intervention immédiate aux consultations à heure et à date fixes, ils se conformaient à une conception du temps propre à la culture, que les informateurs généraux décrivaient ainsi : "c'est tout de suite ou jamais ».
Certains informateurs généraux étaient d'avis que la jeune génération de femmes algonquines a plus tendance à prendre en main ses choix de vie, contrairement aux générations précédentes. De plus, si la femme algonquine se sent bien dans sa vie de tous les jours, elle sera plus encline à faire des achats santé et à choisir des aliments sains, nutritifs et équilibrés. Par ailleurs, certaines informatrices-clés ont dit être plus à l'aise avec l'infirmière provenant d'une Première nation, qui les questionnait régulièrement sur leur consommation de drogues et d'alcool, sur leur soutien familial, etc. L'approche holistique de cette infirmière concordait avec les valeurs des femmes des deux communautés.

Même si les études antérieures ont trouvé que les activités éducatives ne sont pas toujours adaptées à la culture des Premières nations, selon les informatrices-clés, les professionnels de la santé ont facilité l'adoption des comportements de santé et ont organisé plusieurs activités culturellement adaptées aux femmes algonquines, tout comme Daniel et Messer ${ }^{26}$ le recommandent.

\section{Réaliser des économies grâce à une meilleure alimentation}

Après avoir appris comment prendre en charge leur diabète, plusieurs femmes ont réalisé des économies en s'alimentant mieux. Certaines ont affirmé qu'elles fréquentaient moins les restaurants ou qu'elles avaient modifié leur comportement lorsqu'elles mangeaient au restaurant : « J'allais moins au restaurant. Ça me coûtait moins cher au restaurant parce que je coupais mes portions » (Isabelle, ligne 1062). Après leur grossesse, les femmes ont maintenu les comportements de santé qu'elles avaient adoptés, en raison des économies associées à une meilleure alimentation.

Durant leur grossesse, quelques participantes ont eu l'occasion de prendre part à des cuisines collectives financées par le Programme canadien de nutrition prénatale ${ }^{29}$. Elles y ont appris à lire les circulaires et à faire une liste d'épicerie, ainsi que le coût des différents aliments : «Souvent, je regardais les circulaires. [...] 
Je me dirigeais où il $\mathrm{y}$ a des places, où c'est plus en spécial que d'autres places là » (Céline, ligne 1110). Elles affirmaient pouvoir maintenant mieux choisir, en connaissance de cause, la qualité et la quantité des aliments, ce qui entraînait un impact réel sur leur budget.

\section{Accès à ses données de glycémie grâce au glucomètre}

Le dernier thème porte sur la possibilité de contrôler sa glycémie grâce à la technologie. Plusieurs participantes des deux communautés utilisaient un glucomètre pour mesurer leur glycémie : " Ça permet de savoir si... ton sang est normal et tout ça! [...] Tu le vois tout de suite [quand] ton sang est normal » (Céline, ligne 182). Certaines participantes, actuellement non diabétiques, continuaient d'utiliser régulièrement leur glucomètre pour surveiller leur glycémie, à titre préventif.

La technologie accessible dans les centres de santé des deux communautés à l'étude permettait aussi aux femmes algonquines de surveiller et de comparer plus facilement leurs données de glycémie, en téléchargeant les données du glucomètre pour les visualiser sous forme graphique, ce qui leur évitait d'avoir à les noter manuellement. Cette auto-surveillance peut diminuer le recours à des soins cliniques et professionnels et augmenter le sentiment d'indépendance et de confiance en soi, deux éléments importants dans l'autogestion du diabète ${ }^{30}$.

\section{Limites de l'étude}

La présente étude offre une perspective sur les facteurs culturels qui contribuent au maintien des comportements de santé auprès d'une population des Premières nations. La perspective présentée est principalement celle des femmes algonquines qui ont reçu des services éducatifs et du soutien relatifs au DG, pendant leur grossesse et en post-partum. Des informateurs généraux d'origine algonquine et non algonquine ont confirmé les thèmes qui ont émergé des données recueillies. Bien qu'en étudiant les communautés de Lac Simon et de Pikogan nous avons ciblé environ un quart de la population algonquine du Québec ${ }^{31}$, nos résultats pourraient ne pas être représentatifs des femmes algonquines vivant dans des communautés urbaines.

\section{Conclusions}

Malgré son caractère exploratoire, la présente étude permet de conclure que les pratiques culturelles pourraient influencer le maintien des comportements de santé chez les femmes algonquines ayant été atteintes d'un DG.

Notre étude a trouvé qu'une approche centrée sur la famille et les amis, plutôt que sur l'individu, pourrait aider les femmes algonquines ayant été atteintes d'un DG à maintenir des comportements de santé.

L'adaptation culturelle de l'éducation à la santé représentait un facteur essentiel au maintien des comportements de santé chez les participantes à l'étude. Les professionnels de la santé pourraient donc continuer à développer des activités adaptées sur le plan culturel à l'intention des femmes algonquines, par exemple ayant lieu dans les bois ou dans des environnements favorisant l'activité physique. En travaillant en étroite collaboration avec leurs patientes, les professionnels de la santé pourraient leur permettre d'exploiter au maximum leurs capacités, d’être plus autonomes et d'améliorer leur mieux-être ${ }^{32}$. Par ailleurs, Santé Canada a publié un nouveau Guide alimentaire canadien, dont un est destiné spécifiquement aux Premières nations, aux Inuits et aux Métis ${ }^{23}$. Cette version adaptée du Guide pourrait être utilisée afin d'expliquer la valeur nutritionnelle, sociale et spirituelle des aliments traditionnels, et les effets néfastes des aliments transformés, dans le cadre d'une stratégie de réduction de l'obésité et des maladies chroniques associées à l'obésité, comme le diabète ${ }^{33}$. Les femmes algonquines de l'étude ont également réalisé qu'elles pouvaient faire des économies en s'alimentant mieux.

Nos résultats indiquent que le recours à un glucomètre pour surveiller la glycémie semble aider les participantes, ce qui porte à croire que cet outil pourrait faire partie des stratégies d'autogestion préventive. Cette conclusion est cependant contraire aux recommandations de 2009 sur l'emploi du glucomètre de l'Agence canadienne des médicaments et des technologies de la santé $^{34}$, qui n'a pas constaté d'amélioration cliniquement significative des taux d'A1C chez les personnes non insulinodépendantes ${ }^{34}$.

Les thèmes émergeant de la présente étude présentent une perspective culturelle unique qui pourrait aider tant les communautés des Premières nations que les professionnels de la santé à élaborer des stratégies et à mettre en place des services spécifiquement destinés aux femmes algonquines ayant reçu un diagnostic de DG. Ces services et stratégies pourraient contribuer à la santé et au mieux-être des femmes enceintes et de leurs enfants, de même qu'à la prévention du diabète de type 2 dans la population algonquine.

\section{Remerciements}

Les auteures tiennent à remercier les personnes ayant contribué au recrutement des participantes à l'étude : Suzanne Paré, infirmière (Centre de santé de Pikogan), Karen Morency, nutritionniste (Centre de santé de Lac Simon) et Rose Dumont, infirmière (Centre de santé de Lac Simon). Nous tenons aussi à souligner la contribution collective des communautés à l'étude, de même que les contributions individuelles décrites dans la présente étude.

Nous aimerions remercier les organismes suivants pour leur contribution financière reçue sous forme de bourses d'études : Santé Canada, Tembec, la Fondation nationale des réalisations autochtones et l'Ordre régional des infirmières et infirmiers de la Montérégie. De plus, nous remercions Santé Canada, employeur de l'une des auteures au moment de la rédaction de l'article. Merci également au Conseil de bande Abitibiwinni d'octroyer une allocation scolaire pour aider les jeunes algonquins à poursuivre leurs études. 


\section{Annexes}

ANNEXE A

Guide d'entrevue (inspiré de Taylor et collab. ${ }^{14}$, p. 9)

Vision du monde

- Décrivez votre santé actuelle.

- Décrivez ce que vous pensez de votre santé.

- Qu'est-ce que vous faites pour vous maintenir en santé?

- Avez-vous des inquiétudes par rapport à votre santé?

- À votre avis, quels sont les principaux problèmes de santé des femmes autochtones?

- Qu'est-ce qui vous vient à l'esprit lorsque je vous parle de diabète gestationnel?

- Qu'est-ce qui arrive lorsqu'une femme a un diabète gestationnel?

- Et, après sa grossesse?

- Qu'est-ce qui arrive au bébé d'une femme qui a un diabète gestationnel?

- Et, après sa naissance?

- Qu'est-ce qu'une femme de votre communauté fait lorsqu'elle reçoit un diagnostic de diabète gestationnel?

- Dans votre communauté, comment explique-t-on le diabète gestationnel?

- Croyez-vous qu'il s'agit d'un problème de santé plus ou moins fréquent que dans une autre communauté?

- Dans votre communauté, comment soigne-t-on une femme qui a reçu un diagnostic de diabète gestationnel?

Langage

- Quels sont les mots utilisés pour parler du diabète?

- Comment les intervenants de la santé s'expriment-ils pour vous parler de diabète?

Facteurs technologiques

- Pendant votre grossesse, comment effectuiez-vous la surveillance de votre taux de sucre?

- Quelle technologie est utile lorsqu'une femme est atteinte d'un diabète gestationnel?

Facteurs religieux et philosophiques

- Pour votre diabète gestationnel, avez-vous déjà eu recours à des soins ou des traitements d'un sage ou d'un shaman? Pour quelles raisons?

- En quels traitements avez-vous confiance pour soigner le diabète gestationnel?

Facteurs familiaux et sociaux

- Quel est le rôle des personnes de la famille en ce qui concerne le diabète gestationnel?

- Comment sont prises les décisions concernant les soins du diabète gestationnel dans votre famille? Dans votre communauté?

Valeurs culturelles et mode de vie

- Quelle est la cause du diabète gestationnel, selon vous?

- Pourquoi pensez-vous que toutes les choses énumérées causent le diabète gestationnel?

- Quelle a été votre réaction en apprenant que vous aviez un diabète gestationnel?

- Quels changements avez-vous faits pendant votre grossesse lorsque vous avez appris que vous aviez un diabète gestationnel?

- Quels sont les changements que vous n'avez jamais faits et qui vous ont été demandés?

- Qu'est-ce qu'il vous reste de ces changements?

- Quelle est votre plus grande crainte à la suite d'un diabète gestationnel?

- Quelle est pour vous une bonne alimentation ou une alimentation équilibrée?

- Dans votre communauté, quels sont les aliments traditionnels? Font-ils partie de façon courante de l'alimentation de votre communauté?

- À votre avis, l'alimentation traditionnelle peut-elle prévenir un diabète ou un diabète gestationnel?

- Quels sont les aliments qu'une femme qui a un diabète gestationnel doit manger durant sa grossesse?

- Dans votre communauté, est-ce qu'une femme qui a un diabète gestationnel peut faire de l'exercice durant sa grossesse?

Facteurs politiques et légaux

* Comment se préoccupe-t-on des femmes qui ont un diabète gestationnel dans la communauté?

* Qu'est-ce que le Conseil de bande ou les gouvernements pourraient faire pour aider les femmes qui ont un diabète gestationnel?

Facteurs économiques

- Quelles sont les principales dépenses qui ont été occasionnées par le diabète gestationnel?

- Ces dépenses ont-elles eu des répercussions sur votre budget?

Facteurs éducationnels

- Comment avez-vous appris ce que vous savez sur le diabète gestationnel?

- Où trouvez-vous votre information sur le diabète?

- Dans quelle mesure êtes-vous satisfaite des renseignements reçus sur le diabète gestationnel? Comment ces renseignements étaient-ils adaptés à votre culture?

Suite page suivante 


\section{ANNEXE A (Suite) \\ Guide d'entrevue (inspiré de Taylor et collab. ${ }^{14}$, p. 9)}

\section{Pratiques de soins}

- Quels sont les comportements de santé qui vous semblent les plus importants pour maintenir votre santé?

- Comment en êtes-vous venue à associer la santé avec ces comportements?

- À votre avis, est-ce que ces comportements sont aussi importants pour votre (vos) enfant(s)?

- Quels sont les comportements de santé que vous aimeriez transmettre à votre (ou vos) enfant(s)?

- Comment vous y prenez-vous pour les transmettre?

- À votre avis, ces comportements ont-ils un impact sur le diabète ou le diabète gestationnel?

- Si oui, quel impact?

- Si non, quels comportements de santé pourraient avoir un impact?

- À votre avis, quelles sont les actions qu'une femme qui a eu un diabète gestationnel peut effectuer pour tenter de prévenir ou retarder un diabète?

ANNEXE B

Stratégies employées pour assurer la rigueur de l'analyse des données

\begin{tabular}{|c|c|c|}
\hline Critère & Définition ${ }^{17}$ & Stratégies \\
\hline \multirow[t]{2}{*}{ Crédibilité } & \multirow[t]{2}{*}{ Exactitude, véracité et authenticité des résultats } & Transcription textuelle \\
\hline & & Analyse phrase par phrase \\
\hline Confirmation & Approbation des résultats par les informateurs & Soumission des résultats aux informateurs et modification d'un thème \\
\hline $\begin{array}{l}\text { Signification } \\
\text { en contexte }\end{array}$ & $\begin{array}{l}\text { Le sens donné aux résultats reflète celui attribué par des personnes } \\
\text { provenant d'un contexte particulier }\end{array}$ & $\begin{array}{l}\text { Description riche et dense du contexte, appuyée par les notes dans le } \\
\text { journal de bord }\end{array}$ \\
\hline $\begin{array}{l}\text { Modèles } \\
\text { récurrents }\end{array}$ & $\begin{array}{l}\text { Examen approfondi des données pour découvrir les répétitions de } \\
\text { thématiques, de modèles et de comportements reflétant une tendance }\end{array}$ & Entente interjuges \\
\hline Saturation & $\begin{array}{l}\text { Les résultats répondent à l'objectif de recherche et des données } \\
\text { supplémentaires n'ajouteront rien à la compréhension du phénomène }\end{array}$ & $\begin{array}{l}\text { Confirmation par les informateurs que le phénomène était couvert } \\
\text { adéquatement }\end{array}$ \\
\hline \multirow[t]{2}{*}{ Transférabilité } & \multirow{2}{*}{$\begin{array}{l}\text { Application des résultats à d'autres contextes, situations } \\
\text { ou cultures }\end{array}$} & Conférence donnée à Lac-Rapide \\
\hline & & $\begin{array}{l}\text { Présentation à la Direction de la santé des Premières nations et des } \\
\text { Inuits (région du Québec) }\end{array}$ \\
\hline
\end{tabular}

\section{Références}

1. Comité d'experts des Lignes directrices de pratique clinique de l'Association canadienne du diabète. Lignes directrices de pratique clinique 2008 de l'Association canadienne du diabète pour la prévention et le traitement du diabète au Canada. Can J Diabetes. 2008;32(suppl. 2):S1-S225.

2. Le diabète au Canada [Internet]. $2^{\mathrm{e}}$ éd. Ottawa (Ont.) : Santé Canada; 2002 [Consultation le 2 novembre 2010]. Consultable en ligne à la page : http:// www.phac-aspc.gc.ca/publicat/dic-dac2/ francais/01cover-fra.php

3. Lowdermilk DL, Perry S, Bobak IM. Soins infirmiers: périnatalité. Laval : Groupe Beauchemin Éditeur Inc.; 2003.
4. Masseboeuf N, Corset E. Diabète gestationnel, nécessité d'une éducation diététique. Soins. 2002;667:17-9.

5. Boivin S, Derdour-Gury H, Perpetue J, Jeandidier N, Pinget $M$. Diabète et grossesse. Ann Endocrinol (Paris). 2002;63(5):480-7.

6. Rodrigues S, Robinson E, Gray-Donald K. Prevalence of gestational diabetes mellitus among James Bay Cree women in Northern Quebec. CMAJ. 1999;160(9):1293-7.

7. Setji TL, Brown AJ, Feinglos MN. Gestational diabetes mellitus. Clinical Diabetes. 2005;23(1):17-24.

8. Leininger M, McFarland MR. Transcultural nursing: concept, theories, research and practice. $3^{\mathrm{e}}$ éd. Toronto (Ont.) : McGraw-Hill Medical Publishing Division; 2002.
9. Leininger M, McFarland MR. Culture care diversity and universality: a worldwide theory of nursing. $2^{\mathrm{e}}$ éd. Boston (MA) : Jones and Bartlett; 2006.

10. McFarland MR. The ethnonursing research method and the culture care theory: implication for clinical nursing practice. Dans : Parker M, dir. Nursing theories and nursing practice. Philadelphia (PA) : F.A. Davis Company; 2001. p. 377-90.

11. Roy B. Le diabète chez les autochtones : regard sur la situation à Betsiamites, Natashquan et La Romaine. Recherches amérindiennes au Québec. 1999;XXIX(3):3-18. 
12. Roy B. Sang sucré, pouvoirs codés et médecine amère. Diabète et processus de construction identitaire : Les dimensions socio-politiques du diabète chez les Innus de Pessamit [thèse de doctorat], [Sainte-Foy (Qc)] : Université Laval; 2002.

13. Smith-Morris CM. Reducing diabetes in Indian Country: lessons from the three domains influencing Pima diabetes [Internet] Hum Org. 2004;63(1):34-46.

14. Taylor C, Keim KS, Sparrer A, Van Delinder J, Parker S. Social and cultural barriers to diabetes prevention in Oklahoma American Indian women. Prev Chronic Dis. 2004;1(2):1-10.

15. Travers KD. Le recours à la recherche qualitative pour mieux comprendre les origines socioculturelles du diabète chez les Micmacs du Cap Breton. Maladies chroniques au Canada. 1995;16(4).

16. Fortin MF. Le processus de la recherche : de la conception à la réalisation. VilleMont-Royal (Qc) : Décarie Éditeur Inc; 1996.

17. Leininger MM. Theory of culture care diversity and university. Dans : Parker M, dir. Nursing theories and nursing practice. Philadelphia (PA) : F.A. Davis Company; 2001. p. 361-76.

18. Bienvenue aux profils des Premières nations [Internet]. Gatineau (Qc) : Affaires autochtones et Développement du Nord Canada; 2008 [Page mise à jour le 14 novembre 2008; consultation le 19 novembre 2008]. Consultable en ligne à la page : http://pse5-esd5.ainc-inac.gc.ca/fnp/

19. Profils des communautés de 2006 [Internet]. Ottawa (Ont.) : Statistique Canada; 2009 [Consultation le 20 avril 2008]. Consultable en ligne à la page : http://www12.statcan.ca/ census-recensement/2006/dp-pd/prof/ 92-591/index.cfm?Lang $=\mathrm{F}$

20. Miles MB, Huberman, MA. Analyse des données qualitatives. Paris (France) : De Boeck Université; 2003.

21. Conseil de recherches en sciences humaines du Canada, Conseil de recherches en sciences naturelles et en génie du Canada, Instituts de recherche en santé du Canada. Énoncé de politique des trois Conseils : Éthique de la recherche avec des êtres humains [Internet]. Ottawa
(Ont.) : Gouvernement du Canada; 2010 [Consultation le 2 mars 2010]. PDF (64 Ko) téléchargeable à partir du lien : http:// www.ger.ethique.gc.ca/pdf/fra/eptc2/ EPTC_2_FINALE_Web.pdf

22. Devault A, Fréchette L. Le soutien social : ses composantes, ses effets et son insertion dans les pratiques sociosanitaires. Dans : Carroll G, dir. Pratiques en santé communautaire. Montréal (Qc) : Chenelière Éducation; 2006. p. 141-52.

23. Santé Canada. Bien manger avec le Guide alimentaire canadien - Premières Nations, Inuit et Métis [Internet]. Ottawa : Gouvernement du Canada, 2007 [Consultation le 24 novembre 2008]. Consultable en ligne à la page : http:// www.hc-sc.gc.ca/fn-an/food-guide-aliment/ fnim-pnim/index-fra.php

24. Tom-Orme L. Transcultural nursing and health care among Native American peoples. Dans : Leininger M, McFarland MR, dir. Transcultural nursing: concept, theories, research and practice. $3^{\text {e }}$ éd. Toronto (Ont.) : McGraw-Hill Medical Publishing Division; 2002. p. 429-40.

25. American Diabetes Association. Diagnostic and classification of diabetes mellitus. Diabetes Care. 2008;31(suppl. 1):S55-S60. DOI:10.2337/dc08-S055.

26. Daniel M, Messer LC. Perceptions de la gravité de la maladie et des obstacles à l'autogestion de sa santé : prédicteurs de l'équilibre de la glycémie chez les Autochtones atteints de diabète de type 2 . Maladies chroniques au Canada. 2002;23(4):147-56.

27. Quill TE, Brody H. Physician recommendations and patient autonomy: finding a balance between physician power and patient choice. Ann Intern Med. 1996;125(9):763-9.

28. Macaulay AC, Cargo M, Bisset S, Delormier $\mathrm{T}$, Lévesque L, Potvin L, et collab. Community empowerment for the primary prevention of type 2 diabetes: Kanien'keha:ka (Mohawk) ways for the Kahnawake Schools Diabetes Prevention Project. Dans : Ferreira MK, Lang GC, dir. Indigenous peoples and diabetes: community empowerment and wellness. Durham (NC) : Carolina Academic Press;
2006. p. 407-33.

29. Agence de la santé publique du Canada. Programme canadien de nutrition prénatale : Une décennie de promotion de la santé des mères, des bébés et des communautés [Internet]. Ottawa (Ont.) : Gouvernement du Canada; 2007 [Consultation le 29 décembre 2008] [ $\mathrm{n}^{\circ}$ de catalogue : HP10-11/2007]. PDF (3,2 Mo) téléchargeable à partir du lien : http://www.phac-aspc.gc.ca/hp-ps/ dca-dea/publications/pdf/mb_f.pdf

30. Direction générale de la promotion et des programmes de la santé, Santé Canada. Soutenir l'auto-soin : la contribution des infirmières et des médecins - une étude exploratoire. Ottawa (Ont.) : Santé Canada; 1997.

31. Secrétariat aux affaires autochtones. Statistiques des populations autochtones du Québec [Internet]. Québec : Gouvernement du Québec; [Consultation le 26 septembre 2005]. Consultable en ligne à la page : http://www.autochtones.gouv.qc.ca/ nations/population.htm

32. Hagan L, Proulx S. L'éducation pour la santé : le temps d'agir. Infirm Que. 1996:3(3);44-52.

33. Gouvernements fédéral, provinciaux et territoriaux du Canada; les organisations autochtones nationales. Plan directeur pour la santé des Autochtones : Plan décennal de transformations. 24-25 novembre 2005 [Internet]. [Consultation le 24 novembre 2008]. PDF (84 Ko) téléchargeable à partir du lien : http://www.hc-sc.gc.ca/ hcs-sss/alt_formats/hpb-dgps/pdf/ pubs/2005-blueprint-plan-abor-auto/ plan-fra.pdf

34. SCPUOM. Rapport sommaire : Prescription et utilisation optimales des bandelettes de test dans l'autosurveillance glycémique [Internet]. Ottawa (Ont.) : Agence canadienne des médicaments et des technologies de la santé; 2009 [Consultation le 8 octobre 2011]. PDF (1,1 Mo) téléchargeable à partir du lien : http://www.cadth.ca/media/pdf/C1109_ bgts_summary_report_f.pdf 